\title{
Intramural Abscesses: A Sequela of Infective Endocarditis in a Patient with HIV
}

\author{
Chin $\mathrm{CA}^{1}$ and Guo L ${ }^{\star 2}$
}

${ }^{1}$ Schulich School of Medicine and Dentistry, Western University, Richmond St, London, ON, Canada

${ }^{2}$ Department of Cardiac Surgery, University Hospital, Windermere Road, London, ON, Canada

*Corresponding author: Guo L, MD FRCSC, Department of Cardiac Surgery, Room B6-112, University Hospital, 339 Windermere Road, London, ON, Canada N6G 2V4, Fax: (519) 663-3477, Tel: (519) 663-3468, E-mail: linrui.guo@lhsc.on.ca

Citation: Chin CA, Guo L (2016) Intramural Abscesses: A Sequela of Infective Endocarditis in a Patient with HIV. J Case Rep Stud 4(4): 403. doi: 10.15744/2348-9820.4.403

Received Date: April 14, 2016 Accepted Date: August 27, 2016 Published Date: August 30, 2016

\begin{abstract}
Infective Endocarditis (IE) is associated with significant morbidity and mortality, with in-hospital mortality having been reported as ranging from 19-26\%. Commonly documented complications of IE include valvular and chordae destruction and resultant regurgitations, embolic events, peri-valvular abscesses, fistulae, and congestive heart failure. Herein, we report a rare case who presented with embolic myocardial infarction, prosthetic valve IE along with peri-aortic abscess, left ventricular aneurysm and multiple mural abscess with eventual rupture.
\end{abstract}

Keywords: Aneurysm and Rupture; Computed Tomography; Echocardiography; Heart Failure; Infective Endocarditis; Intramural Abscess; Human Immunodeficiency Virus; Myocardial Infarction

List of Abbreviations: CABG: Coronary Artery Bypass Graft; CT: Computed Tomography; ECMO: Extracorporeal Membrane Oxygenation; HIV: Human Immunodeficiency Virus; IE: Infective Endocarditis; LAD: Left Anterior Descending Artery; LV: Left Ventricle; PVE: Prosthetic Valve Endocarditis; TEE: Trans-esophageal Echocardiogram; HAART: Highly Active Anti-Retroviral Therapy

\section{Introduction}

Infective Endocarditis (IE) is a well-known, dangerous condition associated with many varied complications [1-3]. One such complication is peri-valvular abscesses, which affects many patients with prosthetic valve endocarditis (PVE) [4]. Intramural abscesses, however, are much rarer as there is paucity of such reports in the literature. Furthermore, we were unable to find any reports of mortality rates with respect to IE with intramural abscesses involvement.

Endocarditis is often diagnosed using the modified Duke criteria and can have devastating consequences. It affects approximately 10-15 thousand people per year in the United States [5]. Valvular heart disease (e.g. mitral or aortic regurgitation), rheumatic fever, or having a prosthetic valve is risk factors associated with IE. Injection drug use is also important to remember. Approximately $96 \%$ of patients present with the non-specific sign of a fever, and half that (48\%) present with a new murmur. The classic signs of splinter hemorrhages, Janeway lesions, Osler nodes, and Roth spots, appear less frequently, in approximately 8, 5, 3, and 2 percent of patients, respectively [6].

While Gram positive bacterial caused most IE, Enterococcal Endocarditis has increased in incidence over the last decade, and antimicrobial-resistance enterococci are increasingly of concern [7].

There is evidence to suggest that HIV's impact on the clinical course of HIV-positive cardiovascular patients is fortunately diminishing in the Highly Active Anti-retroviral Therapy (HAART) era [8-11]. It should be noted, however, that its associate comorbidities such as diabetes or renal failure may impact clinical course of infective endocarditis in HIV-positive patients [12].

\section{Case Report}

A 66-year old homosexual male underwent the modified Bentall procedure with St. Jude mechanical valve conduit, hemi-arch replacement and single coronary artery bypass graft (CABG; left internal thoracic artery to obtuse marginal artery) for bicuspid aortic valve stenosis, aortapathy, and coronary artery disease in 2012. He had Human Immunodeficiency Virus-positive (HIV+) status since 1996 and had begun anti-retroviral therapy in 2006. At the time of presentation in 2012, his viral load was undetectable, and his CD4 cell count was 560/ $\mathrm{mm}^{3}$. Other co-morbidities included dialysis-dependent chronic renal insufficiency, Type II Diabetes Mellitus, hypertension and hypercholesterolemia. 
The patient did well until he presented with 2 month history of progressive lethargy, weakness and anorexia and severe acute chest pain required emergency admission to the hospital in March 2014. Coronary angiogram showed total occlusion of the left anterior descending artery (LAD) and anterior myocardial infarction. Blood cultures later revealed Enterococcus faecalis and transesophageal echocardiogram (TEE) showed prosthetic aortic valve endocarditis with peri-valvular abscess. The patient exhibited signs of progressive congestive heart failure and thus urgent repeat Bentall procedure, and single CABG with a saphenous vein to his LAD, was carried out.

Intraoperative TEE showed an akinetic anterior wall and apex indicating left ventricular (LV) aneurysm and abscess cavities around the prosthetic aortic valve and annulus. Intra-operatively, multiple intramural abscess cavities were identified and frank pus was drained during the dissection for the LAD. The pus subsequently grew Enterococcus faecalis. The cavities were repaired with multiple sutures. His LAD was not graftable and coronary bypass was abandoned. Infected valve conduit was removed and periannular abscess was debrided and patched with bovine pericardium. A \#21 St. Jude mechanical valve conduit was re-implanted.

After coming off bypass, however, the abscessed anterior ventricular wall ruptured, necessitating a return to bypass for further debridement and repairs. The patient was taken off bypass a second time, with inotropic support and intra-aortic balloon pump.

Post-operatively, he was in cardiogenic shock and was placed on Extracorporeal Membrane Oxygenation (ECMO) due to severe metabolic acidosis. The acidosis persisted despite 2 days of ECMO therapy. Repeat TEE showed very poor left ventricular function and a completely akinetic anterior wall. With no improvement by post-operative day 3 , the family decided to withdraw care and the patient expired.

\section{Discussion}

\section{Extent of the Disease}

The reported incidence of peri-annular abscess in prosthetic valve endocarditis (PVE) cases ranges from 56-100\% [4]. The current case is unusual because of its extent and complexity, with: PVE, peri-aortic valvular abscesses, intramural abscess in a left ventricular aneurysm, embolic myocardial infarction of the left anterior descending artery, and eventual rupture of the abscessed wall. Of particular interest in this case was the presence of multiple intramural abscess formations, which is a very rare complication and no reports in the literature. However, we were certain that these cavities were due to abscess as frank pus was drained and the same strain of bacterium was grown.

As a complication of bacterial endocarditis, intramural abscess is an extremely rare occurrence, but this may also suggest underdiagnosis. In this case, intramural abscess was an incidental intraoperative finding and was not diagnosed with preoperative images. The incidence and mechanism of intramural abscess formation is unknown, but it is conceivable that bacteria or emboli lodge in the small branches of coronary arteries causing vessel occlusion, myocardial necrosis and formation of abscess [13,14]. It may also be from a direct seeding from infected thrombus in the LV aneurysm.

\section{Intramural Abscess as a Prognostic Feature}

Peri-valvular abscesses in endocarditis have been proposed as a predictor for "poor prognosis for patients with endocarditis" [15]. Fistula formation, pericarditis, heart block, and embolic problems are some sequelae of abscesses [3,15]. To the contrary, Chu et al. followed 267 patients short-term, of whom 28 developed abscesses. 21 survived while 7 died. This resulted in a calculated Odds Ratio of 1.52 (95\% CI 0.61 - 3.80; p-value of 0.525) for those with abscesses versus those without [1]. We would be remiss, however, if we ignored the fact that the patient's ventricle ruptured at the site of the intramural abscesses and he suffered an embolic myocardial infarction from this abscess.

\section{Utility of Echocardiography and Computed Tomography in Diagnosing Endocarditis}

Earlier detection of abscesses should improve clinical outcome. The Duke Criteria are useful in diagnosing endocarditis. This case highlights the role of echocardiographic evidence, a "Major" Duke criterion. Indeed, TEE pre-operatively demonstrated the patient's PVE as well as peri-valvular abscesses. However, in this case TEE failed to diagnose the intramural aneurysm and abscess pre-operatively. Still, TEE represents an improvement over Transthoracic Echocardiography (TTE). In their review of echocardiography in IE, Evangelista and Gonzalez-Alujas reported the sensitivity and specificity of TEE for PVE vegetation to be as high as $86-94 \%$ and $88-100 \%$, respectively, versus TTE's sensitivity for PVE of $36-69 \%$ [16].

Enhanced Gated Computed Tomography (CT) is another imaging modality of potential use. Feuchtner et al. reported 9 of 37 patients in their study population were intra-operatively found to have abscess and/or pseudoaneurysms. CT was able to detect all of these. He presented other encouraging data showing CT detected IE in 28 of 29 patients in whom valve abnormalities were detected by TEE) [17]. CT may provide supplementary information to that from TEE, the first-line imaging modality. Unfortunately, in this case, preoperative contrasted CT chest demonstrated neither perivalvular nor intramural abscess likely due to their sizes. However, it would have been helpful to visualize the intramural abscesses and non-graftable LAD before operating. Moreover, there was a possibility that surgery would have been delayed, so as to better plan, or declined by the surgical team. In some cases, it is prudent to consider aborting large-scale, complex operations which may not benefit the patient. 


\section{Impact of HIV/Immune Status on Clinical Course}

It is reasonable to consider whether or not the patient's immune status contributed to his clinical course. There is evidence to suggest that when the CD4 count of a patient with endocarditis is $<200$ cells $/ \mathrm{mm}^{3}$, the patient may be at an increased risk of mortality [18]. In their review involving 1,239 HIV+ patients, Polanco et al. recently concluded that "in contemporary practice HIV does not appear to be associated with incremental operative mortality, except in patients with clinical disorders related to their HIV status" [8]. Vasudev et al. (2016) [9] provided evidence recently in support of the idea that, in the HAART era, the influence of HIV on clinical course and mortality is limited or negligible for patients presenting with IE. Further, complications following cardiovascular surgery are similar between HIV-positive and HIV-negative patients [10,11]. This patient did not present with any HIV-related illness, nor was his CD4 count $<200$ cells $/ \mathrm{mm}^{3}$. Therefore, it appears unlikely that the immune status (CD4 count $=560$ cells $/ \mathrm{mm}^{3}$ when last measured) played a significant role in his demise.

Enterococcus faecalis: This patient's IE was due to Enterococcus faecalis (E. faecalis), which are responsible for $10 \%$ of all IE cases [6]. The source of infection of IE is often through a genitourinary route (e.g. via catheterization or cystoscopy), with the gastrointestinal tract and cutaneous routes being another common portals of entry via infection, malignancy, or inflammation [19]. But the patient denied such a history. Though impossible to be confirmed, it was logical to think that his potential homosexual activities could provide such an entry.

\section{Conclusion}

Enterococcus faecalis endocarditis is a life-threatening disease with a high mortality and is increasingly complicated to treat. Intramural abscesses in endocarditis can present with unique complications that clinicians need to be mindful of in the decision making process.

\section{References}

1. Chu VH, Cabell CH, Benjamin DK Jr, Kuniholm EF, Fowler VG Jr. et al. (2004) Early predictors of in-hospital death in infective endocarditis. Circulation 109: $1745-9$.

2. Hase R, Otsuka Y, Yoshida K, Hosokawa N (2015) Profile of infective endocarditis at a tertiary-care hospital in Japan over a 14-year period: characteristics, outcome and predictors for in-hospital mortality. Int J Infect Dis 33: 62-6.

3. Longo DL, Kasper DL, Jameson JL, Fauci AS, Hauser SL, et al. (2012) Infective Endocarditis In: Harrison's Principles of Internal Medicine (18 ${ }^{\text {th }}$ Edn) USA: McGraw-Hill publisher, USA.

4. Blumberg EA, Karalis DA, Chandrasekaran K, Wahl JM, Vilaro J, et al. (1995) Endocarditis-associated paravalvular abscesses. Do clinical parameters predict the presence of abscess? Chest 107: 898-903.

5. Bayer AS (1993) Infective Endocarditis. Clin Infect Dis 17: 313-20.

6. Murdoch DR, Corey GR, Hoen B, Miró JM, Fowler VG Jr, et al. (2009) Clinical presentation, etiology, and outcome of infective endocarditis in the 21st century: the International Collaboration on Endocarditis-Prospective Cohort Study. Arch Intern Med 169: 463-73.

7. Slipczuk L, Codolosa JN, Davila CD, Romero-Corral A, Yun J, et al. (2013) Infective endocarditis epidemiology over five decades: a systematic review. PLoS One 8: e82665.

8. Polanco A, Itagaki S, Chiang Y, Chikwe J (2014) Changing prevalence, profile, and outcomes of patients with HIV undergoing cardiac surgery in the United States. Am Heart J 167: 363-8.

9. Vasudev R, Shah P, Bikkina M, Shamoon F (2016) Infective Endocarditis in HIV. Int J Cardiol 214: 216-7.

10. Robich MP, Schiltz N, Johnston DR, Mick S, Tse W, et al. (2014) Outcomes of patients with human immunodeficiency virus infection undergoing cardiovascular surgery in the United States. J Thorac Cardiovasc Surg 148: 3066-73.

11. Horberg MA, Hurley LB, Klein DB, Follansbee SE, Quesenberry C, et al. (2006) Surgical outcomes in human immunodeficiency virus-infected patients in the era of highly active antiretroviral therapy. Arch Surg 141: 1238-45.

12. Begezsán II, Bejan C, Ghibu L, Dorobăt CM, Roşu F (2012) Infective endocarditis in non-HIV immunosuppressed patients. Rev Med Chir Soc Med Nat Iasi 116: 687-91.

13. Atik FA, Campos VG, da Cunha CR, de Oliveira FB, Otto ME, et al. (2015) Unusual mechanism of myocardial infarction in prosthetic valve endocarditis. Int Med Case Rep J 8: 111-6.

14. Jenny BE and Almanaseer Y (2014) Aortic valve endocarditis complicated by ST-elevation myocardial infarction. Tex Heart Inst J 41: 668-70.

15. Chan KL (2002) Early clinical course and long-term outcome of patients with infective endocarditis complicated by perivalvular abscess. CMAJ 167: 19-24.

16. Evangelista A, Gonzalez-Alujas MT (2004) Echocardiography in infective endocarditis. Heart 90: 614-7.

17. Feuchtner GM, Stolzmann P, Dichtl W, Schertler T, Bonatti J, et al (2009) Multislice computed tomography in infective endocarditis: comparison with transesophageal echocardiography and intraoperative findings. J Am Coll Cardiol 53: 436-44.

18. Ribera E, Miró JM, Cortés E, Cruceta A, Merce J, et al. (1998) Influence of human immunodeficiency virus 1 infection and degree of immunosuppression in the clinical characteristics and outcome of infective endocarditis in intravenous drug users. Arch Intern Med 158: 2043-50.

19. Dahl A, Bruun NE (2013) Enterococcus faecalis infective endocarditis: focus on clinical aspects. Expert Rev Cardiovasc Ther 11: 1247-57. 


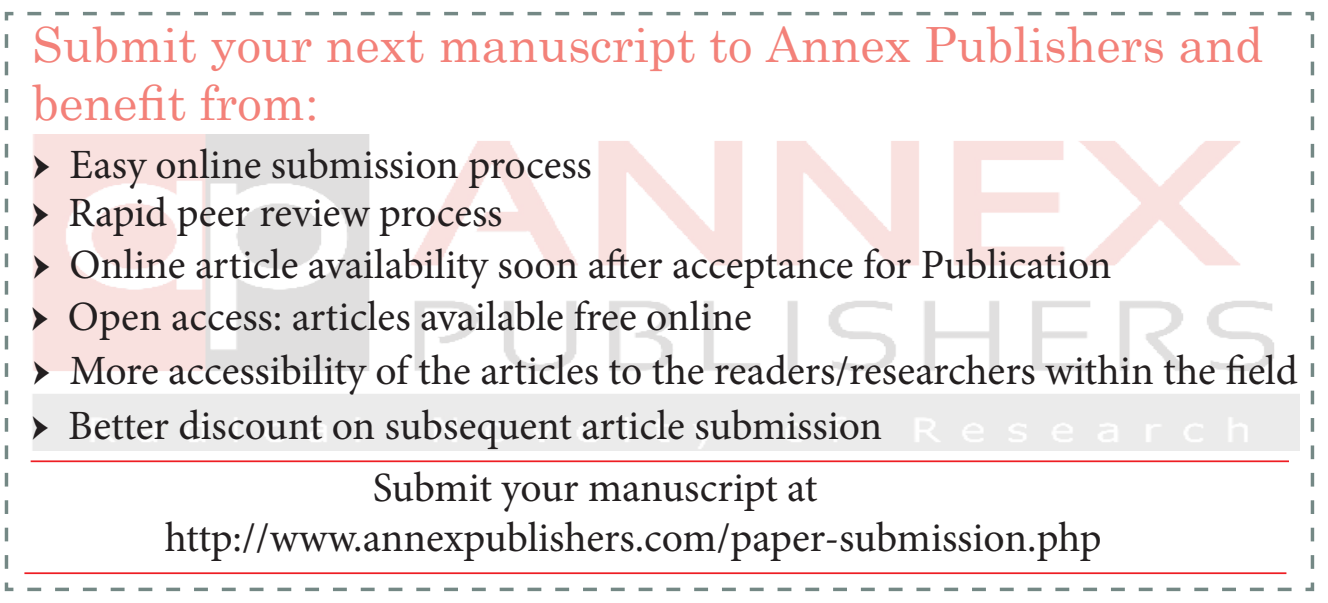

